\title{
Towards the Assignment for the Ground Scalar Meson
}

\author{
Xue-Chao Feng, Peng Chen, and Ji-Min Shang \\ Department of Technology and Physics, Zhengzhou University of Light Industry, Zhengzhou 450002, China
}

Correspondence should be addressed to Xue-Chao Feng; fxchao@zzuli.edu.cn

Received 5 November 2012; Revised 24 December 2012; Accepted 24 December 2012

Academic Editor: C. Q. Geng

Copyright (C) 2013 Xue-Chao Feng et al. This is an open access article distributed under the Creative Commons Attribution License, which permits unrestricted use, distribution, and reproduction in any medium, provided the original work is properly cited.

\begin{abstract}
Combining three complementary approaches, nonrelativistic constituent quark model, Regge phenomenology, and meson-meson mixing, we obtain the mass relations which relate different meson multiplets. With the help of known meson masses, these relations can be used for the prediction of unknown meson states masses. The utility of this new analysis technique is applied for the scalar meson and results in a possible allocation scheme for the ground scalar meson.
\end{abstract}

\section{Introduction}

It is well known that the strong interactions are described by the non-Abelian gauge field theory quantum chromodynamics (QCD). However, we are still far from deriving the spectrum of mesons from first principles so far. Therefore, the phenomenological analysis of meson states is important for the hadronic physics [1-3].

The study of scalar mesons plays an important role in particle physics. The scalar mesons with vacuum quantum numbers could provide a full understanding of the symmetry breaking mechanisms in QCD and the confinement [4]. Moreover, scalar mesons are very important intermediate state in Goldstone boson interactions away from threshold, where chiral perturbation theory is not applicable [5]. Therefore, the investigation of scalar mesons becomes an attention focus in theory and experiments [5-13]. Nevertheless, the interpretation of the nature of scalar mesons is a longstanding problem of particle physics to this day. According to the new edition of Particle Data Group (PDG), for the light quark sector, the number of resonances found in experiment below $2 \mathrm{GeV}$ exceeds the number of states that conventional quark models can accommodate [14]. There are ten resonances are observed in the experiments, including the isoscalar resonances $f_{0}(600), f_{0}(980), f_{0}(1370), f_{0}(1500)$, and $f_{0}(1710)$, the isovector resonances $a_{0}(980)$, and $a_{0}(1450)$, and the isodoublet resonances $K_{0}^{*}(1430), K_{0}^{*}(800)$, and $K_{0}^{*}(1950)$. Up to now, none of the ten reported scalar states can be definitely assigned as ground scalar nonet members.
Considering that some resonances cannot be explained by the conventional $q \bar{q}$ quark model, different theoretical models have also been established such as $K \bar{K}$ molecule [15], MIT bag model [16], QCD sum rules [17], and chiral Lagrangians [18]. For the heavy-light quark meson, two resonances, $D_{S J}^{*}(2317)$ and $D_{0}^{*}(2400)$, are observed in the experiments. The resonances $D_{S J}^{*}(2317)$ with a mass $2317.8 \pm 0.6 \mathrm{MeV}$ and width $349.3 \pm 0.6 \mathrm{MeV}$ are assigned as charm-strange meson of scalar state. However, the experimental value is considerably smaller than the results in many theoretical predictions $[19,20]$. The disagreement results in the different interpretations about the $D_{S J}^{*}(2317)$ such as the molecule state [21], the four-quark state [22], or the $D \pi$ atom [23]. On the other hand, considering the fact that the mass $D_{S J}^{*}(2317)$ is smaller than its partner $D_{0}^{*}(2400)$, we also argue that the assignment of the $D_{S J}^{*}(2317)$ needs further tests in the experiments.

In the present work, based on the relations derive from three complementary approaches, nonrelativistic constituent quark model, Regge phenomenology, and meson-meson mixing, we investigate the scalar meson mass spectrum and propose a possible assignment for the ground scalar meson nonet.

\section{Nonrelativistic Constituent Quark Model for Scalar Meson}

In the framework of the non-relativistic constituent quark model, one typically assumes that the $q \bar{q}$ wave functions are 
a solution of a non-relativistic Schrödinger equation with the Breit-Fermi Hamitonian $H$ [24-26], which contain

$$
\begin{aligned}
H \psi_{n}(r)=( & m_{q}+m_{\bar{q}}+\frac{m_{q}+m_{\bar{q}}}{2 m_{q} m_{\bar{q}}} \overrightarrow{p^{2}}-\left(\frac{m_{q}^{3}+m_{\bar{q}}^{3}}{8 m_{q}^{3} m_{\bar{q}}^{3}}\right) \overrightarrow{p^{4}} \\
& \left.+V_{N R}(r)+H_{L S+S S+T}\right) \psi_{n}(r)
\end{aligned}
$$

with

$$
\begin{gathered}
V_{N R}(r)=V_{v}(r)+V_{s}(r), \\
H_{L S+S S+T}=H_{L S}+H_{S S}+H_{T},
\end{gathered}
$$

where $m_{q}$ and $m_{\bar{q}}$ are the constituent quark masses, $V_{N R}$ is the confining potential consisting of vector, and scalar contributions, $H_{L S}, H_{S S}$, and $H_{T}$, are the spin-spin, spinorbit, and tensor terms, respectively. Following, we apply the Breit-Fermi Hamitonian to the S-wave and P-wave meson multiplets.

For the S-wave mesons, consider the fact the matrix element of the tensor and spin-orbit interactions vanishes, one arrive at the following relation:

$$
M=m_{q}+m_{\bar{q}}+a_{0}+e_{0} \frac{\left\langle S_{q} S_{\bar{q}}\right\rangle}{m_{q} m_{\bar{q}}} .
$$

$S_{q}$ and $S_{\bar{q}}$ are the constituent quark spins, and $e_{0}$ is the spin-spin interaction coefficient. The constant $a_{0}$ is small compared to the constituent quark masses $m_{q}$ and $m_{\bar{q}}$ and may be absorbed into the constituent quark masses [24, 29, 30]. Therefore, the relation (3) can be expressed as

$$
M=m_{q}+m_{\bar{q}}+e_{0} \frac{\left\langle S_{q} S_{\bar{q}}\right\rangle}{m_{q} m_{\bar{q}}} .
$$

For the $P$-wave mesons, the phenomenological form of the matrix element of the Breit-Fermi Hamitonian has the following relation:

$$
\begin{aligned}
M= & m_{q}+m_{\bar{q}}+a_{1}+b_{1}\left(\frac{1}{m_{q}}+\frac{1}{m_{\bar{q}}}\right) \\
& +c_{1}\left(\frac{1}{m_{q}^{2}}+\frac{1}{m_{\bar{q}}^{2}}\right)+\frac{d_{1}}{m_{q} m_{\bar{q}}} \\
& +g_{1}\left[\frac{\left(m_{q}+m_{\bar{q}}\right)^{2}+2 m_{q} m_{\bar{q}}}{4 m_{q}^{2} m_{\bar{q}}^{2}}\langle L \cdot S\rangle\right. \\
& \left.-\frac{m_{q}^{2}-m_{\bar{q}}^{2}}{4 m_{q}^{2} m_{\bar{q}}^{2}}\left\langle L\left(S_{q}-S_{\bar{q}}\right)\right\rangle\right] \\
& +e_{1} \frac{\left\langle S_{q} S_{\bar{q}}\right\rangle}{m_{q} m_{\bar{q}}}+f_{1}\left(\frac{1}{m_{q}^{3}}+\frac{1}{m_{\bar{q}}^{3}}\right)+h_{1} \frac{a(j, 1)}{m_{q} m_{\bar{q}}}
\end{aligned}
$$

with

$$
\begin{gathered}
a(j, 1)=\frac{4}{5}\left\langle S^{2} L^{2}-\frac{3}{2}(L S)-3(L S)^{2}\right\rangle, \\
\langle L S\rangle=\frac{1}{2}[j(j+1)-3 S-2],
\end{gathered}
$$

where $a_{1}, b_{1}, c_{1}, d_{1}, e_{1}, f_{1}, g_{1}$, and $h_{1}$ are the constants, and the angular momentum parts of the matrix elements are shown in Table 1. With the help of the parameters in Table 1, from the relations (4) and (5), we obtain the following formulas:

$$
\begin{gathered}
\frac{M_{\pi}+3 M_{\rho}}{2 M_{K}+6 M_{K^{*}}-M_{\pi}-3 M_{\rho}}=\frac{m_{n}}{m_{s}}, \\
\frac{M_{s \bar{s}}\left(1^{3} P_{2}\right)-M_{s \bar{s}}\left(1^{3} P_{0}\right)}{M_{n \bar{n}}\left(1^{3} P_{2}\right)-M_{n \bar{n}}\left(1^{3} P_{0}\right)}=\left(\frac{m_{n}}{m_{s}}\right)^{2},
\end{gathered}
$$

where $M_{n \bar{n}}$ is the mass of isovector state, $M_{s \bar{s}}$ refers to the mass of the bare strange member of the isoscalar states, and $m_{s}$ and $m_{n}$ ( $n$ stands for nonstrange $u$ - and $d$-quark) are the corresponding constituent quark masses. In view of relation (8), we can obtain the scalar mesons masses from the masses of other meson states, which quark model assignment is firmly established. Due to the isovector meson act as a beacon for the mass scales of meson nonet, we should firstly determine the mass of isovector state. In the following section, we are going to calculate the mass of isovector states in the framework of Regge phenomenology and mass mixing matrix.

\section{Mass Mixing Matrix of Isoscalar States}

In the quark model, mesons are $q \overline{q^{\prime}}$ bound states of quark $q$ and antiquark $\overline{q^{\prime}}$. Three light flavors of quarks $-u,-d$, and $-s$ combined with three antiquarks yield nine meson nonet, which contain two bare isoscalar states. In general, the two bare isoscalar states can mix, which result in two physical isoscalar states. Therefore, the mix of isoscalar states is important for the meson, due to providing a clue for the assignment of a resonance in a meson nonet.

In the nonstrange $N=(u \bar{u}+d \bar{d}) / \sqrt{2}$ and strange $S=s \bar{s}$ basis, the mass-squared matrix describing the mixing of the two physical isoscalar states can be written as [31]

$$
M^{2}=\left(\begin{array}{cc}
M_{N}^{2}+2 A & \sqrt{2} A X \\
\sqrt{2} A X & M_{s \bar{s}}^{2}+A X^{2}
\end{array}\right),
$$

where $M_{N}$ and $M_{s \bar{s}}$ are the masses of bare states $N$ and $S$, respectively; $A$ are the mixing parameters which describe the $q \bar{q} \leftrightarrow q^{\prime} \bar{q}^{\prime}$ transition amplitudes. The $X$ that is a phenomenological parameter describes the $S U(3)$ broken ratio of the nonstrange ( $u$ - and $d$-quark) and strange quark propagators via the constituent quark mass ratio. The value of $X$ is determined to be 0.63 by inserting the corresponding masses into relation (7) [29]. Here and below, all the masses used as input for our calculation are taken from PDG [14].

In the meson nonet, the physical isoscalar states $\varphi$ and $\varphi^{\prime}$ are the eigenstates of mass-squared matrix $M^{2}$, as well 
TABLE 1: Angular momentum part of the matrix elements of relations (4) and (5).

\begin{tabular}{lcccc}
\hline$n^{2 s+1} l_{J}$ & $1^{1} S_{0}$ & $1^{3} S_{1}$ & $1^{3} P_{2}$ & $1^{3} P_{0}$ \\
\hline$\langle L S\rangle$ & & 1 & -2 \\
$a(j, 1)$ & & $-2 / 5$ & -4 \\
$\left\langle S_{q} S_{\bar{q}}\right\rangle$ & $-3 / 4$ & $1 / 4$ & $1 / 4$ & $1 / 4$ \\
$\left\langle L\left(S_{q}-S_{\bar{q}}\right)\right\rangle$ & & 0 & 0 \\
\hline
\end{tabular}

TABLE 2: Predicted mass of bare $s \bar{s}$ for meson nonet. $\theta_{\text {quad }}$ is the nonet mixing angle from the quadratic mass formulae [14].

\begin{tabular}{ccccccc}
\hline & Nonet & $1^{1} S_{0}$ & $1^{3} S_{1}$ & $1^{3} P_{2}$ & $1^{3} D_{3}$ & $2^{1} S_{0}$ \\
\hline$M_{s \bar{s}}(\mathrm{MeV})$ & Present work & 685 & 1018 & 1530 & 1858 & 1323 \\
& {$[27]$} & 697 & 1009 & 1546 & & \\
& {$[28]$} & 702 & 1010 & 1538 & & \\
& {$[19]$} & 960 & 1020 & 1530 & 1900 & 1630 \\
$\theta_{\text {quad }}\left({ }^{\circ}\right)$ & & -11.5 & 38.7 & 29.6 & 32 & -22.4 \\
\hline
\end{tabular}

as the square masses $M_{\varphi}^{2}$ and $M_{\varphi^{\prime}}^{2}$ are the eigenvalues, respectively. The physical states $\varphi$ and $\varphi^{\prime}$ can be related to the $s \bar{s}$ and $N=(u \bar{u}+d \bar{d}) / \sqrt{2}$ by

$$
\left(\begin{array}{l}
\text { phy }_{1} \\
\text { phy }_{2}
\end{array}\right)=U\left(\begin{array}{c}
N \\
S
\end{array}\right)
$$

and the unitary matrix $U$ can be described as

$$
U M^{2} U^{\dagger}=\left(\begin{array}{cc}
M_{\mathrm{phy}_{1}}^{2} & 0 \\
0 & M_{\mathrm{phy}_{2}}^{2}
\end{array}\right) .
$$

The mix of the physical isoscalar states can be also described as

$$
\left(\begin{array}{l}
\mathrm{phy}_{1} \\
\mathrm{phy}_{2}
\end{array}\right)=\left(\begin{array}{cc}
\cos \theta & -\sin \theta \\
\sin \theta & \cos \theta
\end{array}\right)\left(\begin{array}{l}
\varphi_{8} \\
\varphi_{1}
\end{array}\right)
$$

with

$$
\begin{aligned}
\varphi_{1} & =\frac{(u \bar{u}+d \bar{d}+s \bar{s})}{\sqrt{3}}, \\
\varphi_{8} & =\frac{(u \bar{u}+d \bar{d}-2 s \bar{s})}{\sqrt{6}},
\end{aligned}
$$

where $\theta$ is the nonet mixing angle.

From the relations (9), (10), (11), and (12), one will obtain

$$
U=\left(\begin{array}{cc}
\cos \theta & -\sin \theta \\
\sin \theta & \cos \theta
\end{array}\right)\left(\begin{array}{cc}
\sqrt{\frac{1}{3}} & -\sqrt{\frac{2}{3}} \\
\sqrt{\frac{2}{3}} & \sqrt{\frac{1}{3}}
\end{array}\right)
$$

Based on the relations (9) and (11), one can have the following relations:

$$
\begin{aligned}
M_{N}^{2}+2 A= & \left(\sqrt{\frac{1}{3}} \cos \theta-\sqrt{\frac{2}{3}} \sin \theta\right)^{2} M_{\text {phy }_{1}}^{2} \\
& +\left(\sqrt{\frac{2}{3}} \cos \theta+\sqrt{\frac{1}{3}} \sin \theta\right)^{2} M_{\text {phy }_{2}}^{2}, \\
M_{s \bar{s}}^{2}+A X^{2}= & \left(\sqrt{\frac{1}{3}} \cos \theta-\sqrt{\frac{2}{3}} \sin \theta\right)^{2} M_{\mathrm{phy}_{2}}^{2} \\
& +\left(\sqrt{\frac{2}{3}} \cos \theta+\sqrt{\frac{1}{3}} \sin \theta\right)^{2} M_{\text {phy }_{1}}^{2}
\end{aligned}
$$

Applying the relations (15) for the meson states $1^{1} S_{0}, 1^{3} S_{1}$, $1^{3} P_{2}, 1^{3} D_{3}$, and $2^{1} S_{0}$, we obtain the bare $s \bar{s}$ member masses, and the results are present in Table 2 . Here and below, all the masses and the mixing angle used as input for our calculation are taken from the PDG [14].

\section{Regge Phenomenology and Mass of Isovector Scalar Meson}

Regge phenomenology is widely used for the calculation of the meson and baryonium spectrum [28, 36-38]. In [28], $\mathrm{Li}$ et al. indicate that the quasi-linear Regge trajectories could provide a reasonable description for the meson mass spectrum. Anisovich et al. also conclude that the meson states can fit to the quasi-linear Regge trajectories at the $\left(N, M^{2}\right)$ and $\left(J, M^{2}\right)$ planes with good accuracy [36]. In the last decade, Regge trajectories are reanalysis to make predictions for the masses of states not yet to be discovered in experiment or to determine the quantum numbers of the newly discovered states. 
TABLE 3: Predicted mass $M_{n \bar{n}}, M_{n \bar{s}}, M_{s \bar{s}}$, and $M_{c \bar{s}}$ of ground scalar meson. (All in MeV and the values used as input for our analysis are shown in boldface.)

\begin{tabular}{|c|c|c|c|c|c|c|c|}
\hline Nonet & Mass & $M_{n \bar{n}}$ & $M_{n \bar{s}}$ & $M_{s \bar{s}}$ & $M_{c \bar{n}}$ & $M_{c \bar{s}}$ & $M_{c \bar{c}}$ \\
\hline \multirow{4}{*}{$1^{3} P_{0}$} & Present work & 1024 & 1279 & 1414 & 2318 & 2423 & 3415 \\
\hline & {$[32]$} & $960 \pm 30$ & $1220_{-150}^{+50}$ & $1220 \pm 40$ & & & \\
\hline & {$[24]$} & $983 \pm 10$ & $1204 \pm 10$ & $1397 \pm 16$ & & & \\
\hline & [19] & 1090 & 1240 & 1360 & 2400 & 2480 & 3440 \\
\hline \multirow{4}{*}{$2^{3} P_{0}$} & Present work & 1611 & 1826 & 1924 & 2835 & 3099 & 3958 \\
\hline & {$[33]$} & & & & 2880 & 2970 & \\
\hline & {$[34,35]$} & & & & 2919 & 3054 & \\
\hline & {$[19]$} & 1780 & 1890 & 1990 & & & 3920 \\
\hline $3^{3} P_{0}$ & Present work & 2034 & 2243 & 2390 & 3270 & 3653 & 4434 \\
\hline
\end{tabular}

Based on the assumption that the hadrons with identical $J^{\text {PC }}$ quantum numbers obey quasi-linear form of Regge trajectories, one have the following relations [28]:

$$
\begin{aligned}
& l=\alpha_{i \overline{i^{\prime}}}(0)+\alpha_{\overline{i i^{\prime}}}^{\prime} M_{\overline{i i^{\prime}}}^{2}, \\
& l=\alpha_{i \overline{j^{\prime}}}(0)+\alpha_{i \overline{j^{\prime}}}^{\prime} M_{\overline{i j^{\prime}}}^{2}, \\
& l=\alpha_{j \overline{j^{\prime}}}(0)+\alpha_{j \overline{j^{\prime}}}^{\prime} M_{\overline{j j^{\prime}}}^{2},
\end{aligned}
$$

where $i \overline{i^{\prime}}$ denotes the quark and antiquark flavors, and $l$ and $M_{i i^{\prime}}$ are the orbital momentum and mass of the $i i^{\prime}$ meson. The parameters $\alpha_{i \bar{i}^{\prime}}^{\prime}$ and $\alpha_{i i^{\prime}}(0)$ are, respectively, the slope and intercept of the Regge trajectory. The intercepts and slopes can be expressed by $[28,39]$

$$
\begin{gathered}
\alpha_{i \bar{i}}(0)+\alpha_{j \bar{j}}(0)=2 \alpha_{i \bar{j}}(0), \\
\frac{1}{\alpha_{i i^{\prime}}^{\prime}}+\frac{1}{\alpha_{j \bar{j} j^{\prime}}^{\prime}}=\frac{2}{\alpha_{i \overline{j^{\prime}}}^{\prime}} .
\end{gathered}
$$

The relation (17) is satisfied in two-dimensional QCD [40], the dual-analytic model [41], and the quark bremsstrahlung model [42]. The relation (18) is deprived from the topological and the $q \bar{q}$-string picture of hadrons [43]. Recently, based on the available data for mesonic resonances of light, medium, and heavy flavors, Filipponi et al. [44] and Burakovsky and Goldman [45] construct different form slopes (19) of linear Regge trajectories for all quark flavors. One has the following:

$$
\begin{aligned}
& \alpha_{i \bar{j}}^{\prime}=\frac{0.9 \mathrm{GeV}^{-2}}{1+0.22\left(\left(m_{i}+m_{j}\right) / \mathrm{GeV}\right)^{3 / 2}}, \\
& \alpha_{i \bar{j}}^{\prime}=\frac{\alpha^{\prime}}{\pi / 4+(\pi / 4) \sqrt{\alpha^{\prime}}\left(m_{i}+m_{j}\right) / 2} .
\end{aligned}
$$

According to the relations (19), we find the slope of Regge trajectory depends on the constituent quark masses through the combination $\left(m_{i}+m_{j}\right)$. The relations (19) are obtained by fitting experimental data, and these relations are possible to update with the increasing amount of experimental data. In the present work, we adopt the assumption presented by $[28,31,46]$ that the slopes of the parity partners (e.g., the $0^{+}$, $1^{-}, 2^{+}$, and $1^{-}$are parity partners) trajectories coincide, and further, that the slopes do not depend on charge conjugation in accordance with the C-invariance of QCD. Under this assumption, the corresponding slopes of the $1^{3} P_{2}$ are the same as those of the $1^{3} P_{0}$ trajectories, and we have the following relations:

$$
\begin{aligned}
1+ & \frac{M^{2}\left(1^{3} P_{0}\right)_{n \bar{n}} M^{2}\left(1^{3} P_{2}\right)_{n \bar{s}}-M^{2}\left(1^{3} P_{0}\right)_{n \bar{s}} M^{2}\left(1^{3} P_{2}\right)_{s \bar{s}}}{M^{2}\left(1^{3} P_{0}\right)_{n \bar{s}} M^{2}\left(1^{3} P_{2}\right)_{s \bar{s}}-M^{2}\left(1^{3} P_{0}\right)_{s \bar{s}} M^{2}\left(1^{3} P_{2}\right)_{n \bar{s}}} \\
& =\frac{4 M^{2}\left(1^{3} P_{0}\right)_{n \bar{n}} M^{2}\left(1^{3} P_{2}\right)_{n \bar{s}}-4 M^{2}\left(1^{3} P_{0}\right)_{n \bar{s}} M^{2}\left(1^{3} P_{2}\right)_{s \bar{s}}}{M^{2}\left(1^{3} P_{0}\right)_{n \bar{n}} M^{2}\left(1^{3} P_{2}\right)_{s \bar{s}}-M^{2}\left(1^{3} P_{0}\right)_{s \bar{s}} M^{2}\left(1^{3} P_{2}\right)_{n \bar{n}}}, \\
1+ & \frac{M^{2}\left(1^{3} P_{0}\right)_{c \bar{c}} M^{2}\left(1^{3} P_{2}\right)_{\bar{n}}-M^{2}\left(1^{3} P_{0}\right)_{c \bar{n}} M^{2}\left(1^{3} P_{2}\right)_{n \bar{n}}}{M^{2}\left(1^{3} P_{0}\right)_{c \bar{s}} M^{2}\left(1^{3} P_{2}\right)_{n \bar{n}}-M^{2}\left(1^{3} P_{0}\right)_{n \bar{n}} M^{2}\left(1^{3} P_{2}\right)_{c \bar{n}}} \\
& =\frac{4 M^{2}\left(1^{3} P_{0}\right)_{c \bar{c}} M^{2}\left(1^{3} P_{2}\right)_{c \bar{n}}-4 M^{2}\left(1^{3} P_{0}\right)_{c \bar{n}} M^{2}\left(1^{3} P_{2}\right)_{n \bar{n}}}{M^{2}\left(1^{3} P_{0}\right)_{c \bar{c}} M^{2}\left(1^{3} P_{2}\right)_{n \bar{n}}-M^{2}\left(1^{3} P_{0}\right)_{n \bar{n}} M^{2}\left(1^{3} P_{2}\right)_{c \bar{c}}} \\
1+ & \frac{M^{2}\left(1^{3} P_{0}\right)_{c \bar{c}} M^{2}\left(1^{3} P_{2}\right)_{c \bar{s}}-M^{2}\left(1^{3} P_{0}\right)_{c \bar{s}} M^{2}\left(1^{3} P_{2}\right)_{\bar{s} \bar{s}}}{M^{2}\left(1^{3} P_{0}\right)_{c \bar{s}} M^{2}\left(1^{3} P_{2}\right)_{s \bar{s}}-M^{2}\left(1^{3} P_{0}\right)_{s \bar{s}} M^{2}\left(1^{3} P_{2}\right)_{c \bar{s}}} \\
& =\frac{4 M^{2}\left(1^{3} P_{0}\right)_{c \bar{c}} M^{2}\left(1^{3} P_{2}\right)_{c \bar{s}}-4 M^{2}\left(1^{3} P_{0}\right)_{c \bar{s}} M^{2}\left(1^{3} P_{2}\right)_{s \bar{s}}}{M^{2}\left(1^{3} P_{0}\right)_{c \bar{c}} M^{2}\left(1^{3} P_{2}\right)_{s \bar{s}}-M^{2}\left(1^{3} P_{0}\right)_{s \bar{s}} M^{2}\left(1^{3} P_{2}\right)_{c \bar{c}}}
\end{aligned}
$$

In relations (20), we eliminate the slopes and obtain masses expressions which relate six meson states. Inserting the masses of the corresponding mesons into relations (20), we obtain the masses of $M_{n \bar{n}}, M_{n \bar{s}}, M_{\bar{s} \bar{s}}$, and $M_{c \bar{s}}$ of ground scalar meson. In our previous work, a series of relations between meson mass and the parameters of radial trajectories are established in the framework of Regge phenomenology $[47,48]$. The relations are still applicable for the scalar meson. Inserting the ground scalar meson masses and the parameters in $[47,48]$, the radial excited meson masses are predicted. The results are compared with other predictions 


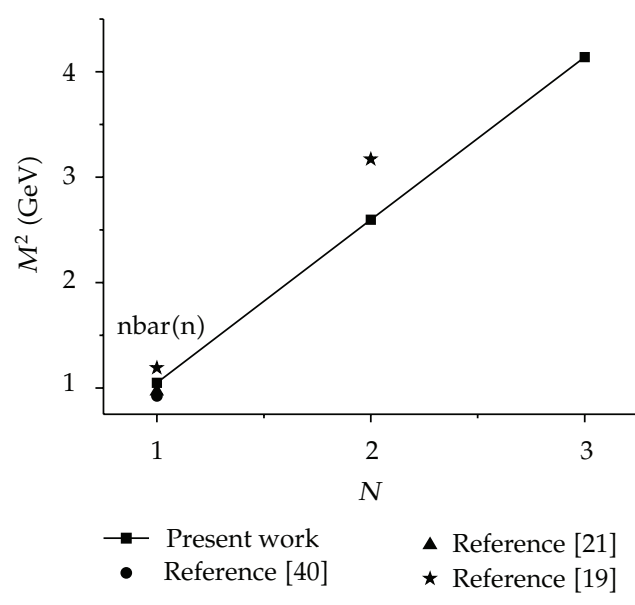

(a)

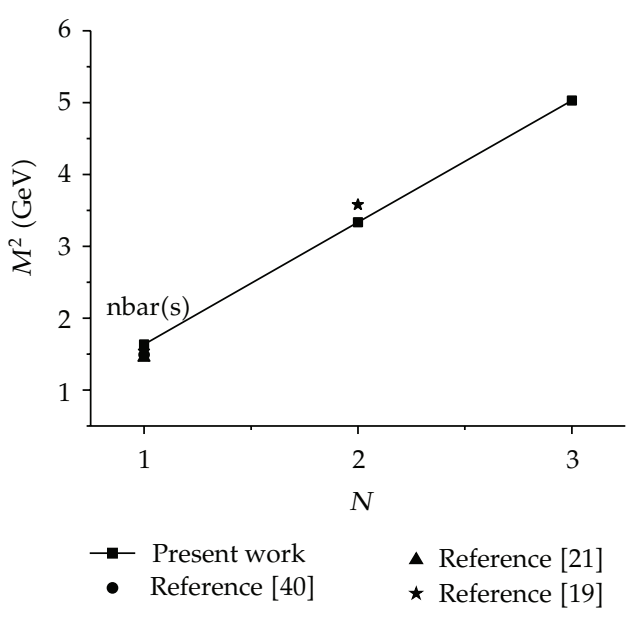

(b)

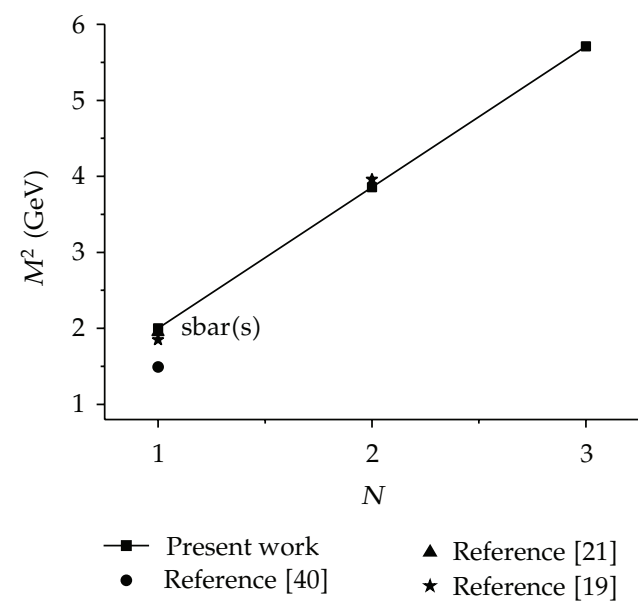

(c)

Figure 1: The quasi-linear trajectory for $N^{3} P_{0}$ meson nonet.

and shown in Table 3. Moreover, the radial excited masses of the light quark sector are shown in Figure 1.

\section{Results and Discussion}

According to the introduction in Section 1, the isovector member of nonet is important for the assignment of isoscalar meson, due to providing a beacon for the mass scale of meson nonet. However, both states the $a_{0}(980)$ and $a_{0}(1450)$ are observed in the experiments and could be considered as candidates. In Table 3, our results favor the interpretation of the state $a_{0}(980)$ rather than the $a_{0}(1450)$ as the isovector ground scalar meson. The assignment is supported by the different theoretical models prediction. In the naive quark model, for $S=1$ states, spin-orbit coupling results in mass order $m\left(2^{++}\right) \geq m\left(1^{++}\right) \geq m\left(0^{++}\right)$, which supports the fact that $a_{0}(980)$ (not the $a_{0}(1450)$ ) is the isovector member of scalar nonet $[49,50]$. In the scheme of K-matrix analysis, Anvisoch also indicate that the mass of the $1^{3} P_{0}$ isovector state is about $960 \pm 30 \mathrm{MeV}$, which is in excellent agreement with our prediction [32].
Apart from the isovector member, the isoscalar sector is also ambiguous, both experimentally and theoretically. In Table 3, we propose the bare nonstrange and strange members masses of isoscalar meson.

In the last decade, the heavy-light mesons that attract more attention with many new resonances have been observed in the experiments, such as $D_{s 0}^{*}(2317), D_{s 1}^{*}(2460)$, $D_{s J}^{*}(2715)$, and $D_{s J}^{*}(2860)$. For the $D_{s 0}^{*}(2317)$, it was first observed in $D_{S}^{+} \pi$ by the Babar Collaboration at the Stanford Linear Accelerator Center with a mass of about $2317 \mathrm{MeV}$ and width of about $350 \mathrm{MeV}$ [51]. Subsequently, this state has also been reported by the Bell Collaboration [52] and the CLEO [53]. In our present work, the mass of the charmstrange meson is about $100 \mathrm{MeV}$ higher than the measured mass of $D_{s 0}^{*}(2317)$, which is consistent with the predictions by Godfrey and Isgur [19]. Consequently, we suggest that the assignment for the $D_{s 0}^{*}(2317)$ as a $1^{3} P_{0}$ charm-strange meson needs further testing in the experiment.

In summary, the understanding of scalar meson is quite puzzling for the theorists and experimentalists. Until now, there is no one state that can be assigned as the scalar 
meson member without dispute. In the present work, combining three complementary approaches, non-relativistic constituent quark model, Regge phenomenology, and mesonmeson mixing, we investigate the assignment of ground scalar meson. Our results are useful for the assignment of the scalar meson in the future.

Moreover, we also find a bridge which relate the heavy and light flavor mesons in the process of analyzing scalar meson. The bridge is significant for the assignment of the discovered states and searching the new resonances which have not been observed in the experiments.

\section{Acknowledgment}

This project supported by the Zhengzhou University of Light Industry Foundation, China (Grant nos. 2009XJJ011 and 2012XJJ008).

\section{References}

[1] A. M. Polyakov, "Interaction of goldstone particles in two dimensions. Applications to ferromagnets and massive YangMills fields," Physics Letters B, vol. 59, no. 1, pp. 79-81, 1975.

[2] P. Maris and C. D. Roberts, "Dyson-schwinger equations: a tool for hadron physics," International Journal of Modern Physics E, vol. 12, no. 3, article 297, 2003.

[3] M. Gell-Mann, "A schematic model of baryons and mesons," Physics Letters, vol. 8, no. 3, pp. 214-215, 1964.

[4] F. E. Close and N. A. Tornqvist, "Scalar mesons above and below 1 GeV," Journal of Physics G, vol. 28, no. 10, article R249, 2002.

[5] A. H. Fariborz, "Isosinglet scalar mesons below $2 \mathrm{GeV}$ and the scalar glueball mass," International Journal of Modern Physics A, vol. 19, no. 13, article 2095, 2004.

[6] Y. H. Liu, D. M. Li, J. X. Shao, X. G. Wang, and Z. Y. Zhang, "Regarding the scalar mesons," Physical Review D, vol. 77, no. 3, Article ID 034025, 5 pages, 2008.

[7] D. M. Li, K. W. Wei, and Y. Hong, "A possible assignment for the ground scalar meson nonet," European Physical Journal A, vol. 25, pp. 263-266, 2005.

[8] M. Schumacher, "Structure of scalar mesons and the Higgs sector of strong interaction," Journal of Physics G, vol. 38, no. 8, Article ID 083001, 2011.

[9] Z. T. Xia and W. Zuo, "A mixing scheme for the structure of $f_{0}(600)$ and $f_{0}(1370)$," Nuclear Physics A, vol. 848, pp. 317-329, 2010.

[10] X. Liu and Z. J. Xiao, "Light scalar mesons and charmless hadronic $\mathrm{B}_{c} \rightarrow$ SP,SV decays in the perturbative QCD approach," Physical Review Letters, vol. 82, Article ID 054029, 2010.

[11] Z. G. Wang, "Analysis of the nonet scalar mesons as tetraquark states with new QCD sum rules," International Journal of Theoretical Physics, vol. 51, no. 2, pp. 507-517, 2012.

[12] A. H. Fariborz, R. Jora, and J. Schechter, "Light scalar puzzle in QCD," AIP Conference Proceedings, vol. 1361, pp. 127-131, 2011.

[13] W. de Paula and T. Frederico, "Scalar mesons within a dynamical holographic QCD model,” Physics Letters B, vol. 693, no. 3, pp. 287-291, 2010.

[14] J. Beringer, J.-F. Arguin, R. M. Barnett et al., "Review of particle physics," Physical Review D, vol. 86, no. 1, Article ID 010001, 2012.
[15] J. Weinstein and N. Isgur, “ $K \bar{K}$ molecules," Physical Review $D$, vol. 41, no. 7, pp. 2236-2257, 1990.

[16] R. L. Jaffe, "Multiquark hadrons. I. Phenomenology of $Q^{2} Q^{-2}$ mesons," Physical Review D, vol. 15, no. 1, pp. 267-280, 1977.

[17] F. Shi, T. G. Steele, V. Elias, K. B. Sprague, Y. Xue, and A. H. Fariborz, "Hölder inequalities and isospin splitting of the quark scalar mesons," Nuclear Physics A, vol. 671, no. 1-4, pp. 416-446, 2000.

[18] D. Black, A. H. Fariborz, S. Moussa, S. Nasri, and J. Schechter, "Unitarized pseudoscalar meson scattering amplitudes from three flavor linear sigma models," Physical Review D, vol. 64, no. 1, Article ID 014031, 45 pages, 2001.

[19] S. Godfrey and N. Isgur, "Mesons in a relativized quark model with chromodynamics," Physical Review D, vol. 32, no. 1, pp. 189-231, 1985.

[20] M. Di Pierro and E. J. Eichten, "Excited heavy-light systems and hadronic transitions," Physical Review D, vol. 64, no. 11, Article ID 114004, 20 pages, 2001.

[21] T. Barnes, F. E. Close, and H. J. Lipkin, "Implications of a Dk molecule at 2.32-GeV," Physical Review D, vol. 68, Article ID 054006, 5 pages, 2003.

[22] H. Y. Cheng and W. S. Hou, " $B$ decays as spectroscope for charmed four-quark states," Physics Letters B, vol. 566, no. 3-4, pp. 193-200, 2003.

[23] A. P. Szczepaniak, "Description of the $D_{s}^{*}$ resonance as the $D \pi$ atom," Physics Letters B, vol. 567, no. 1-2, pp. 23-26, 2003.

[24] P. V. Chliapnikov, " $S$ - and $P$-wave meson spectroscopy in the nonrelativistic quark model," Physics Letters B, vol. 496, no. 3-4, pp. 129-136, 2000.

[25] W. Lucha, F. F. Schöberl, and D. Gromes, "Bound states of quarks," Physics Reports, vol. 200, no. 4, pp. 127-240, 1991.

[26] D. Flamm and F. Schöberl, Introduction to Quark Model of Elementary Particles, vol. 1, Gordon and Breach Science Publishers, London, UK, 1982.

[27] K. W. Wei, X. P. Dong, and G. Lu, "Masses of $s \bar{s}$ states and nonet mixing angles," International Journal of Modern Physics A, vol. 26, no. 12, article 2065, 2011.

[28] D. M. Li, B. Ma, Y. X. Li, Q. K. Yao, and H. Yu, "Meson spectrum in regge phenomenology," The European Physical Journal C, vol. 37, no. 3, pp. 323-333, 2004.

[29] D. M. Li and Z. Li, "Strange axial-vector mesons mixing angle," The European Physical Journal, vol. 28, pp. 369-373, 2006.

[30] L. Burakovsky and T. Goldman, "Regarding the enigmas of $P$ wave meson spectroscopy," Physical Review D, vol. 57, no. 5, pp. 2879-2888, 1998.

[31] M. M. Brisudova, L. Burakovsky, and T. Goldman, "New glueball-meson mass relations," Physical Review D, vol. 58, no. 11, Article ID 114015, 7 pages, 1998.

[32] A. V. Anisovich and A. V. Sarantsev, "K-matrix analysis of the $K \pi \mathrm{S}$-wave in the mass region $900-2100 \mathrm{MeV}$ and nonet classification of scalar $q \bar{q}$-states ," Physics Letters B, vol. 413, no. 1-2, pp. 137-146, 1997.

[33] A. M. Badalian and B. L. G. Bakker, "Higher excitations of the $D$ and $D_{\text {s }}$ mesons," Physical Review D, vol. 84, no. 3, Article ID 034006, 10 pages, 2011.

[34] D. Ebert, R. N. Faustov, and V. O. Galkin, "Mass spectra and Regge trajectories of light mesons in the relativistic quark model," Physical Review D, vol. 79, no. 11, Article ID 114029, 11 pages, 2009. 
[35] D. Ebert, R. N. Faustov, and V. O. Galkin, "Heavy-light meson spectroscopy and regge trajectories in the relativistic quark model," The European Physical Journal C, vol. 66, no. 1-2, pp. 197-206, 2010.

[36] A. V. Anisovich, V. V. Anisovich, and A. V. Sarantsev, "Systematics of $q \bar{q}$ states in the $\left(n, M^{2}\right)$ and $\left(J, M^{2}\right)$ planes," Physical Review $D$, vol. 62, no. 5, Article ID 051502, 5 pages, 2000.

[37] A. Zhang, "Charmonium spectrum and new observed states," Physics Letters B, vol. 647, no. 2-3, pp. 140-144, 2007.

[38] X. H. Guo, K. W. Wei, and X. H. Wu, "Some mass relations for mesons and baryons in Regge phenomenology," Physical Review D, vol. 78, no. 5, Article ID 056005, 21 pages, 2008.

[39] L. Burakosky and T. Goldman, "On the regge slopes intramultiplet relation," Physics Letters B, vol. 434, no. 3-4, pp. 251-256, 1998.

[40] R. C. Brower, J. Ellis, M. G. Schmidt, and J. H. Weis, "What is the relativistic generalization of a linearly rising potential?" Nuclear Physics B, vol. 128, no. 1, pp. 75-92, 1977.

[41] N. A. Kobylinsky, E. S. Martynov, and A. B. Prognimak, "Interrelations between intercepts of regge trajectories and new mass formulas," Ukrainskii Fizicheskii Zhurnal, vol. 24, pp. 969974, 1979.

[42] V. V. Dixit and L. A. Balazs, "Regge intercepts of multiquark systems in feynman's bremsstrahlung analogy," Physics Letters $D$, vol. 20, no. 3, pp. 816-819, 1979.

[43] A. B. Kaidalov, "Hadronic mass-relations from topological expansion and string model," Zeitschrift für Physik C Particles and Fields, vol. 12, no. 1, pp. 63-66, 1982.

[44] S. Filipponi, G. Pancheri, and Y. Srivastava, "Regge trajectories for all flavors," Physical Review Letters, vol. 80, no. 9, pp. 18381840, 1998.

[45] L. Burakovsky and T. Goldman, "Comment on "regge trajectories for all flavors'”' Physical Review Letters, vol. 82, no. 2, pp. 457-457, 1999.

[46] D. M. Li, B. Ma, and Y. H. Liu, "Understanding the masses of the $c \bar{s}$ states in regge phenomenology," The European Physical Journal C, vol. 51, pp. 359-365, 2007.

[47] Y. H. Liu, X. C. Feng, and J. J. Zhang, "Interpreting the masses of mesons in regge phenomenology," The European Physical Journal, vol. 43, pp. 379-388, 2010.

[48] X. C. Feng, Y. Jia, T. Q. Chang, and J. Y. Li, "Radial excitation mass spectrum of tensor meson nonet," Acta Physica Polonica $B$, vol. 42, no. 1, pp. 25-32, 2011.

[49] L. Maiani, F. Piccinini, A. D. Polosa, and V. Riquer, "Positive parity scalar mesons in the $1-2 \mathrm{GeV}$ mass range," European Physical Journal C, vol. 50, no. 3, pp. 609-616, 2007.

[50] E. Borchi and R. Gatto, "Dynamical theory of nonleptonic hyperon $P$ waves in SU(6) symmetry," Physical Review Letters, vol. 14, no. 13, pp. 507-509, 1965.

[51] B. Aubert et al., "Observation of a narrow meson state decaying to $D_{S}{ }^{+} \pi^{0}$ at a mass of $2.32 \mathrm{GeV} / \mathrm{c}^{2}$," Physical Review Letters, vol. 90, no. 24, Article ID 242001, 7 pages, 2003.

[52] P. Krokovny, K. Abe, T. Abe et al., "Observation of the $\mathrm{D}_{s J}(2317)$ and $\mathrm{D}_{s J}(2457)$ in $B$ decays," Physical Review Letters, vol. 91, no. 26, Article ID 262002, 6 pages, 2003.

[53] D. Besson, S. Anderson, V. V. Frolov et al., "Observation of a narrow resonance of mass $2.46 \mathrm{GeV} / \mathrm{c}^{2}$ decaying to $D_{S}{ }^{*+} \pi^{0}$ and confirmation of the DsJ (2317) state," Physical Review D, vol. 68, no. 3, Article ID 032002, 10 pages, 2003. 

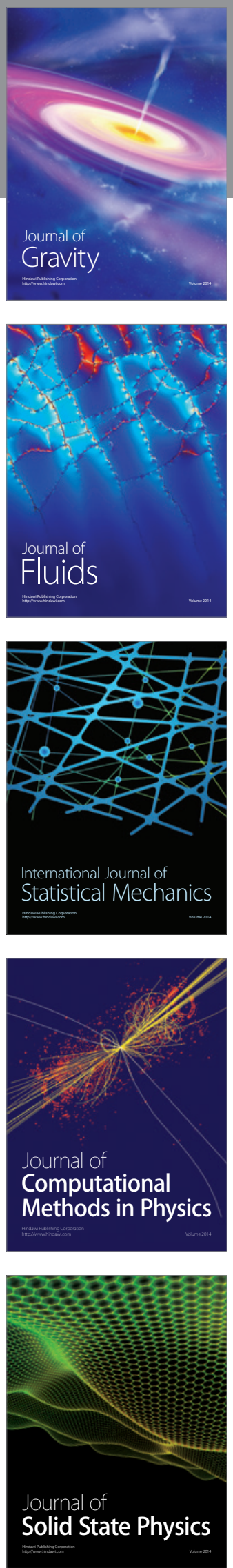



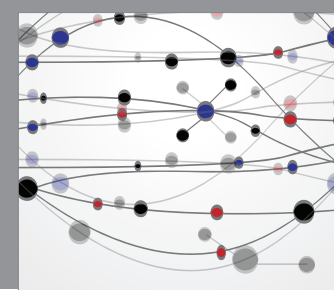

The Scientific World Journal



Soft Matter
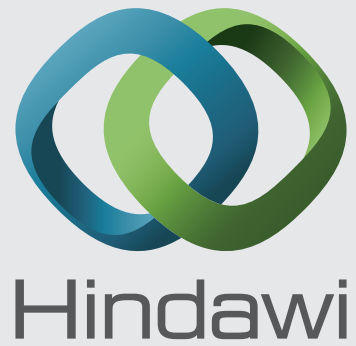

Submit your manuscripts at

http://www.hindawi.com
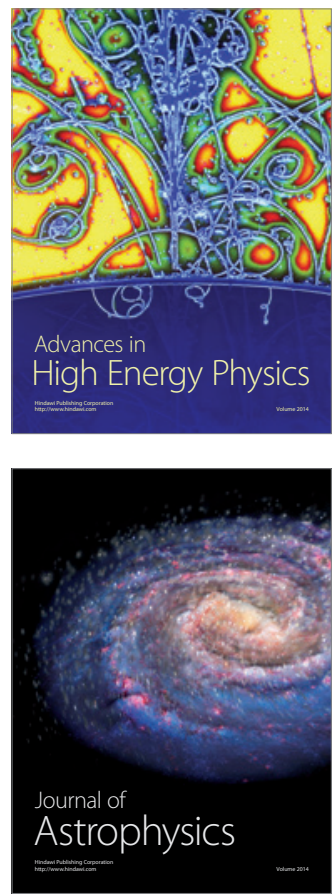
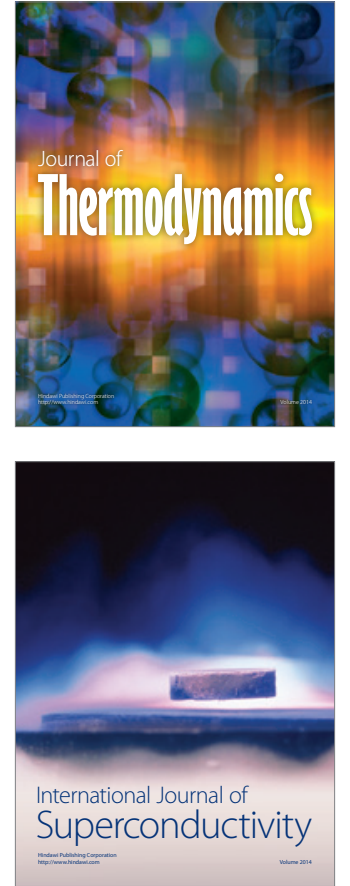
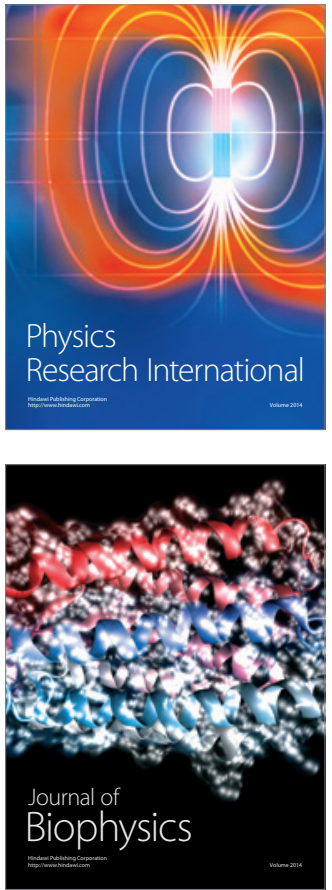
